\title{
Commentary
}

\section{PUZZLING POLYCYSTIN}

\author{
Qais Al-Awqati \\ Departments of Medicine and Physiology, College of Physicians and \\ Surgeons of Columbia University, New York, New York, U.S.A.
}

Adult dominant polycystic kidney disease (ADPKD) is produced by mutations in at least three loci, but the one on chromosome $16 \mathrm{p} 3.3$ (PKD1) accounts for most cases. The identification of the full-length coding sequence of this protein, now named polycystin, represented a tour de force of positional cloning methods, not only because of the protein's size (4300 amino acids), but also because a close region of chromosome 16 contains four duplicated copies of long sequences of PKD1, as well as three other highly homologous genes. The $\mathrm{C}$ terminus of the predicted polycystin sequence has many potential transmembrane loops, whereas the $\mathrm{N}$ terminus contains a signal peptide, two leucine-rich repeats flanked by cysteine regions, and a C-type lectin, making it likely that this region is extracellular. There is an additional motif, termed "PKDl repeats" or "Ig-like repeats," which is composed of 85 amino acids and repeated at least 15 times. Although these structural motifs do not specify a known function, they do imply involvement in adhesive interactions.

Early studies suggested that abnormalities in the extracellular matrix (ECM) are a central problem in ADPKD, since investigators found morphological abnormalities in the basement membrane of cystic tubules. In addition, ADPKD cells, unlike normal kidney epithelial cells, did not form tubules when cultured inside collagen matrices. It was also found that cyst epithelial cells have a higher proliferative rate, with occasional adenomas being seen in the cysts, suggesting that these cells might not be well differentiated. The polarized distribution of the sodium, potassium ATPase was disrupted in some cystlining epithelial cells. Recently, it was found that there was a high number of apoptotic cells in cystic kidney. The mechanism of cyst formation and enlargement is now ascribed to induction or uncovering of an epithelial secretory process mediated by the cystic fibrosis transmembrane rectifier (CFTR) chloride channel (for a review see
Refs. 1 and 2). At present, it is somewhat difficult to tie these observations into a unifying hypothesis.

To begin to link polycystin to these findings, immunocytochemical localization of polycystin is the first logical step, and intriguing results are presented by Palsson et al., in this issue (3), by two other published studies $(4,5)$, and another four in preliminary form (6-9). The results differ among these studies, perhaps because of the use of either anti-peptide or monoclonal antibodies, which might allow recognition of homologous proteins. In addition, the need for performing the studies in human tissue sections introduces a considerable problem of tissue preservation and fixation artifacts. Palsson's antibodies recognize murine polycystin, allowing studies that are free of these concerns. Immunoblots showed a large number of bands in all published studies, but whether these indicate a large number of alternately spliced forms, proteolytic cleavage, or just lack of specificity of these antibodies remains to be determined.

All groups found that polycystin is overexpressed in the epithelial cells of cysts. ADPKD cells overexpress both mutant and wild-type alleles, and Palsson et al. find that the proteins are in intracellular vesicles rather than in the plasma membranes, suggesting that polycystin might interact with another protein in order to reach the surface, perhaps explaining the dominant inheritance of the disease. Palsson and Griffin (5), but not Ward (4), found that polycystin is abundant in embryonic kidney but almost absent in the adult. Immunofluorescence studies using an antibody against the $\mathrm{N}$-terminal region showed that polycystin was present in the plasma membrane of some embryonic tubules, but was localized to the basal surface of the ureteric bud, reminiscent of the staining pattern of extracellular matrix proteins (3). But how does this fit with the many transmembrane domains present in the sequence? There is some evidence that an alterna- 
tively spliced form of polycystin might code for a protein composed only of the extracellular domain $(10,11)$. Of course, a matrix proteinase could also release the extracellular domain into the ECM. Dramatic localization was also seen in the membrane (or matrix) of the cells that form the glomerular crevice of the S-shaped body (see Palsson's Fig. 3a). In later stages, the staining was present in the visceral epithelium of the glomeruli. Others $(4,9)$ have found a different staining pattern in the glomerulus, and future studies will have to resolve this problem.

The most puzzling finding in ADPKD is that cysts arise in only a minority of tubules and, even in these nephrons, epithelial cells in the noncystic areas appear normal. Further, microdissection has shown that affected nephrons contain very few cysts. Assuming that polycystin is expressed only (or largely) during embryonic development, what mechanisms could allow for such a low probability of cyst formations? Polycystin's effect might force cells that are in contact with it to progress through a specific developmental pathway, and its absence could lead to an "instability" in the phenotype. But how is instability produced in only a few cells, when the normal protein is expressed in most of them? Perhaps the polycystin "receptor" can be activated by other ligands whose expression is localized, allowing a few cells to escape their effect. (A formally equivalent hypothesis is intersection of the polycystin signal transduction pathway by activation of other receptors). Although this scenario is plausible, it would still have to explain that cysts can develop in all segments of the nephron, hence the "spurious" ligands have to be expressed at early times of development, when all the progenitors of the different nephron segments are still within a reasonable diffusion distance, leaving only a few cells outside the field of influence of the "spurious" ligands. Another possible interpretation of this "instability" is that ADPKD is formally similar to an epithelial cancer, such as familial adenomatous polyposis, where the mutation is present in all cells but the probability of forming adenomas is rare. In this disease, local factors in the cell (other mutations) or its environment are required to make the mutant cells assume a different phenotype.

The presence of the new molecular reagents should allow identification of the forms of this protein and the functions of the various domains and its receptor, and, more importantly, the development of an assay to generate and test hy- potheses of its role in kidney development is now possible. The good news is that polycystin has turned out to be a protein with an unknown function, raising our hopes that new mechanisms in kidney development will be identified.

\section{REFERENCES}

1. Grantham JJ. (1993) Fluid secretion, cellular proliferation and the pathogenesis of renal epithelial cysts. J. Am. Soc. Nephrol. 3: 1843-1857.

2. Harris PC, Ward CJ, Peral B, Hughes J. (1995) Polycystic kidney disease 1: Identification and analysis of the primary defect. $J$. Am. Soc. Nephrol. 6: 1125-1133.

3. Palsson R, Sharma C, Kim K, McLaughlin M, Brown D, Arnaout MA. (1996) Characterization of Polycsytin, the product of autosomal polycystic kidney disease 1. Mol. Med. 6: 1702-1711.

4. Ward CJ, Tuley H, Ong AC, et al. (1996) Polycystin, the polycystic kidney disease 1 protein, is expressed by epithelial cells in fetal adult and cystic kidneys. Proc. Nat. Acad. Sci. U.S.A. 93: 1524-1528.

5. Griffin MD, Torres VE, Grande JP, Kumar R. (1996) Immunolocalization of polycystin in human tissues and cultured cells. Proc. Assoc. Am. Phys. 108: 185-197.

6. Onuchic L, Wilson P, O'Sullivan E, Hebert R, Germino G. (1995) Polycystin is developmentally regulated and increased in human cystic epithelium. J. Am. Soc. Nephrol. 6: 705. Abstract.

7. van Adelsberg JS. (1995) Immunocytochemical localization and biochemical characterization of the PKDl gene product. J. Am. Soc. Nephrol. 6: 712. Abstract.

8. Geng L, Peissel B, Deng N, et al. (1995) Polycystin, the product of the PKDl gene. J. Am. Soc. Nephrol. 6: 695.

9. Peters DJM, Klinger R, Bernini L, deHeer E, Breuning MH, Bruijn JA. (1995) Immunohistochemical analysis of renal tissue with polyclonal antibodies against peptides encoded by the predicted ADPKD1 protein. $J$. Am. Soc. Nephrol. 6: 706. Abstract.

10. The International Polycystic Disease Consortium. (1995) Polycystic kidney disease: The complete structure of the $P K D I$ gene and its protein. Cell 81: 289-298.

11. Hughes J, Ward CJ, Peral B. (1995) The polycystic kidney disease 1 (PKD1) gene encodes a novel protein with multiple cell recognition domains. Nat. Genet. 10: 151-160. 